\section{Admission levels of high-density lipoprotein and apolipoprotein A-1 are associated with the neurologic outcome in patients with out-of-hospital cardiac arrest}

\author{
Yong Soo Son, Kyung Su Kim, Gil Joon Suh, Woon Yong Kwon, \\ Min Ji Park, Jung In Ko, Taegyun Kim \\ Department of Emergency Medicine, Seoul National University Hospital, Seoul, Korea
}

Objective To investigate whether serum levels of high-density lipoprotein (HDL) and apolipoprotein A-1 (ApoA1), after the return of spontaneous circulation, can predict the neurologic outcome in patients with out-of-hospital cardiac arrest (OHCA).

Methods This was a retrospective observational study conducted in a single tertiary hospital intensive care unit. All adult OHCA survivors with admission lipid profiles were enrolled from March 2013 to December 2015. Good neurologic outcome was defined as discharge cerebral performance categories 1 and 2 .

Results Among 59 patients enrolled, $13(22.0 \%)$ had a good neurologic outcome. Serum levels of HDL (56.7 vs. $40 \mathrm{mg} / \mathrm{dL}$ ) and ApoA1 (117 vs. $91.6 \mathrm{mg} / \mathrm{dL}$ ) were significantly higher in patients with a good outcome. Areas under the HDL and ApoA1 receiver operating curves to predict good outcomes were 0.799 and 0.759 , respectively. The proportion of good outcome was significantly higher in patients in higher tertiles of $\mathrm{HDL}$ and ApoA1 (test for trend, both $\mathrm{P}=0.003$ ). $\mathrm{HDL}$ $(\mathrm{P}=0.018)$ was an independent predictor in the multivariate logistic regression model.

Conclusion Admission levels of $\mathrm{HDL}$ and ApoA1 are associated with neurologic outcome in patients with OHCA. Prognostic and potential therapeutic values of HDL and ApoA1 merit further evaluation in the post-cardiac arrest state, as in other systemic inflammatory conditions such as sepsis.

Keywords Heart arrest; Cholesterol, HDL; Apolipoprotein A-1; Prognosis

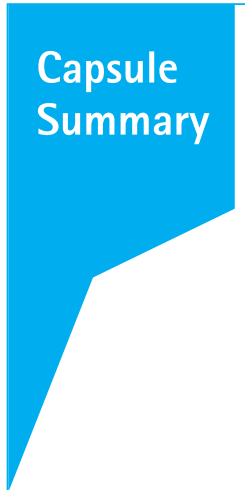

What is already known

It is known that decreased levels of high-density lipoprotein and apolipoprotein A-1 are associated with poor outcome in patients with systemic inflammatory diseases such as sepsis and acute pancreatitis.

What is new in the current study

We show that decreased levels of high-density lipoprotein and apolipoprotein A-1 are associated with poor neurologic outcome in patients with out-of-hospital cardiac arrest.
elSSN: 2383-4625

Received: 15 July 2017

Revised: 3 September 2017

Accepted: 10 November 2017

Correspondence to: Kyung Su Kim Department of Emergency Medicine, Seoul National University Hospital, 101 Daehak-ro, Jongno-gu, Seoul 03080, Korea

E-mail:kanesu@gmail.com

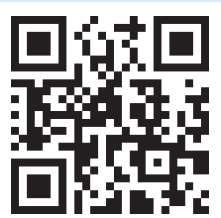

How to cite this article:

Son YS, Kim KS, Suh GJ, Kwon WY, Park MJ, Ko Jl, Kim T. Admission levels of high-density lipoprotein and apolipoprotein A-1 are associated with the neurologic outcome in patients with out-of-hospital cardiac arrest. Clin Exp Emerg Med 2017;4(4):232-237.

This is an Open Access article distributed under the terms of the Creative Commons Attribution Non-Commercial License (http:// creativecommons.org/licenses/by-nc/4.0/). 


\section{INTRODUCTION}

Sudden cardiac death is one of the major causes of death worldwide and overall survival rate of cardiac arrest victims remains unsatisfactory. ${ }^{1,2}$ Even if a return of spontaneous circulation is achieved successfully, patients suffer a global ischemia-reperfusion injury, resulting in multiple organ dysfunction and death. ${ }^{3}$ The post-cardiac arrest state is also referred to as a sepsis-like syndrome, since inflammatory mediators play a pivotal role in this condition. ${ }^{4}$

Classically, high-density lipoprotein (HDL) and its major component, apolipoprotein A-1 (ApoA1) are known to have anti-inflammatory and anti-atherogenic effects and to exert a preventive effect for cardiovascular diseases. ${ }^{5}$ Recent studies have reported that serum HDL and ApoA1 levels measured after admission can be used as a prognostic marker in various critical conditions such as sepsis and pancreatitis, and in the post-operative state. ${ }^{6-9}$ However, there has been no study reporting the prognostic role of HDL and ApoA1 in a post-cardiac arrest state. We hypothesized that $\mathrm{HDL}$ and ApoA1 measured after admission would be low in patients with poor neurologic outcomes because those patients are more likely to have a severe ischemia-reperfusion injury.

The aim of this study is to investigate whether serum levels of HDL and ApoA1 at admission are associated with neurologic outcomes in post-cardiac arrest patients.

\section{METHODS}

\section{Study setting}

This was a retrospective observational study performed in a 12bed emergency intensive care unit of a tertiary referral hospital from March 2013 to December 2015 in South Korea. Out-of-hospital cardiac arrest (OHCA) patients were initially managed by fire station-based emergency medical technicians, and advanced life support was administered in the emergency department. If the patients were successfully resuscitated, they were eligible for admission, and post-cardiac arrest care was delivered, based on the recommendations of the 2010 American Heart Association guidelines. ${ }^{10}$ Therapeutic hypothermia targeting $33^{\circ} \mathrm{C}$ for 24 hours and emergent coronary angiography were performed at the discretion of the attending physician. Neurologic outcome was evaluated using serial neurologic examinations, electroencephalography, and brain imaging. The withdrawal of life-sustaining care was only applicable in brain death patients for the purpose of organ donation. The study was approved by the institutional review board (H-1604-123-756) and exempt from patient informed consent.

\section{Participants and data collection}

Patients who were successfully resuscitated from OHCA and admitted to the emergency intensive care unit were identified. Patients younger than 15 years of age and patients in whom HDL and ApoA1 were not tested within 24 hours of return of spontaneous circulation were excluded.

All data were collected based on a unified definition of variables. Abstracted variables were as follows: (1) demographics (age, sex, body mass index); (2) underlying diseases (hypertension, diabetes); (3) cardiac arrest variables (witnessed, initial cardiac rhythm, presumed cardiac cause); (4) admission serum laboratory findings (albumin, C-reactive protein, creatinine, neuron specific enolase, lactate, total cholesterol, triglycerides, HDL, ApoA1, apolipoprotein $B[A p o B]$ ); (5) hospital care (the application of therapeutic hypothermia, emergent coronary angiography); (6) physiologic severity scores on the day of admission (Acute Physiology and Chronic Health Evaluation [APACHE] II, Sequential Organ Failure Assessment [SOFA] score), and; (7) outcome variables (survival discharge, cerebral performance category [CPC] at hospital discharge).

Total cholesterol, triglycerides, and HDL were measured using TBA 200FR (Toshiba, Tokyo, Japan), and ApoA1 and ApoB were measured using BioMajesty Series JCA-BM6010 (JEOL, Tokyo, Japan).

A good neurologic outcome was defined as a CPC score of 1 or 2 , and a bad neurologic outcome was defined as a CPC score of 3 to 5 .

\section{Statistical analysis}

Continuous variables were expressed as means with 95\% confidence intervals, and categorical variables were presented as the percentage frequency of occurrence. A Student t-test or a Wilcoxon rank-sum test was used to compare continuous variables, and the chi-square test or the Fischer exact test was used to compare binomial variables, as appropriate. The performance of lipid profiles to predict a good outcome was compared, using the area under the receiver operating characteristics curve (AUROC). Patients were divided into tertiles, according to HDL and ApoA1 levels, and a test for trend was performed to evaluate the positive association between HDL/ApoA1 and neurologic outcome. Diagnostic performances of $\mathrm{HDL}$ and ApoA1 for a good outcome at various cutoff values were described, using sensitivity, specificity, positive predictive value, and negative predictive value. Multivariate logistic regression analysis, with backward stepwise selection (significance level 0.1), was performed to test the independent predictive value of $H D L$ and $A p o A 1$, respectively. A two-tailed Pvalue of less than 0.05 was considered to indicate statistical sig- 
nificance. All analyses were performed using Stata ver. 13.1 (StataCorp., College Station, TX, USA).

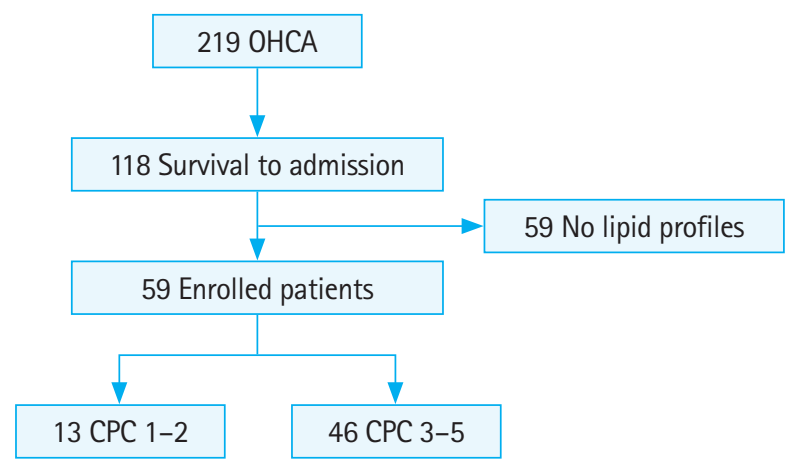

Fig. 1. Patient enrollment. OHCA, out-of-hospital cardiac arrest; CPC, cerebral performance category.

\section{RESULTS}

A total of 219 OHCA patients visited the emergency department during the study period. Among 118 live admissions, 59 patients were enrolled in this study (Fig. 1). Thirteen patients (22.0\%) had a good neurologic outcome (CPC $1[n=3]$ and $2[n=10]$ ) and 46 patients had a poor neurologic outcome (CPC $3[n=9]$, CPC 4 $[n=16]$, CPC $5[n=21])$. The characteristics of the enrolled patients are described in Table 1. The good CPC group was younger and more likely to be female and present more favorably in respect to initial shockable rhythm, cardiac cause, and coronary angiography than the bad CPC group. The good CPC group had lower lactate and C-reactive protein levels, and higher albumin, HDL, and ApoA1 levels. APACHE II and SOFA scores were lower in the good CPC group.

Table 1. Patient characteristics according to neurologic outcomes

\begin{tabular}{|c|c|c|c|c|}
\hline Variable & Total $(n=59)$ & Good CPC $(n=13)$ & Bad CPC $(n=46)$ & P-value \\
\hline \multicolumn{5}{|l|}{ Demographics } \\
\hline Age in years & $57.8(52.9-62.7)$ & $48.9(39.6-58.3)$ & $60.3(54.6-66.0)$ & 0.015 \\
\hline Sex, male & $43(72.9)$ & $6(46.2)$ & $37(80.4)$ & 0.014 \\
\hline Body mass index & $22.3(21.2-23.5)$ & $22.2(20.8-23.5)$ & $22.8(20.5-25.1)$ & 0.798 \\
\hline \multicolumn{5}{|l|}{ Underlying diseases } \\
\hline Hypertension & $19(32.2)$ & $3(23.1)$ & $16(34.8)$ & 0.517 \\
\hline Diabetes & $14(23.7)$ & $1(7.7)$ & $13(28.3)$ & 0.159 \\
\hline \multicolumn{5}{|l|}{ Cardiac arrest variables } \\
\hline Witnessed & $45(76.3)$ & 12 (92.3) & $33(71.7)$ & 0.159 \\
\hline Shockable rhythm & $14(23.7)$ & $8(61.5)$ & $6(13.0)$ & 0.001 \\
\hline Cardiac cause & 19 (32.2) & $11(84.6)$ & $8(17.4)$ & $<0.001$ \\
\hline \multicolumn{5}{|c|}{ Admission serum laboratory results } \\
\hline Lactate $(\mathrm{mmol} / \mathrm{L})$ & $7.2(6.0-8.5)$ & $4.6(1.9-7.2)$ & $8.0(6.6-9.3)$ & 0.006 \\
\hline Albumin $(\mathrm{g} / \mathrm{dL})$ & $3.4(3.2-3.5)$ & $3.9(3.7-4.1)$ & $3.2(3.0-3.4)$ & $<0.001$ \\
\hline C-reactive protein (mg/L) & $4.5(2.6-6.3)$ & $1.2(0-2.6)$ & $5.4(3.1-7.7)$ & 0.025 \\
\hline Creatinine $(\mathrm{mg} / \mathrm{dL})$ & $1.8(1.4-2.3)$ & $1.1(0.8-1.3)$ & $2.1(1.5-2.6)$ & 0.006 \\
\hline NSE $(\mathrm{ng} / \mathrm{mL})^{\mathrm{a})}$ & $57.2(45.8-68.6)$ & $44.2(24.2-64.2)$ & $60.8(47.1-74.5)$ & 0.091 \\
\hline Total cholesterol (mg/dL) & $142.4(129.5-155.4)$ & $156.5(136.0-177.0)$ & $138.5(122.8-154.2)$ & 0.252 \\
\hline Triglycerides (mg/dL) & $153.0(109.4-196.6)$ & $138.9(22.1-255.7)$ & $156.9(109.0-204.9)$ & 0.735 \\
\hline $\mathrm{HDL}(\mathrm{mg} / \mathrm{dL})$ & 43.7 (39.4-47.9) & $56.7(49.2-64.2)$ & $40.0(35.4-44.6)$ & $<0.001$ \\
\hline ApoA1 (mg/dL) & $97.2(88.2-106.2)$ & $117.0(105.5-128.5)$ & $91.6(81.0-102.3)$ & 0.002 \\
\hline ApoB (mg/dL) & $67.9(60.6-75.3)$ & $73.5(59.3-87.6)$ & $66.4(57.7-75.1)$ & 0.426 \\
\hline \multicolumn{5}{|l|}{ Hospital care } \\
\hline Therapeutic hypothermia & $41(69.5)$ & $10(76.9)$ & $31(67.4)$ & 0.735 \\
\hline Coronary angiography & $22(37.3)$ & $9(69.2)$ & $13(28.3)$ & 0.010 \\
\hline \multicolumn{5}{|l|}{ Severity score at admission } \\
\hline APACHE II score & $29.3(27.1-31.5)$ & $24.4(18.6-30.1)$ & $30.7(28.4-33.0)$ & 0.017 \\
\hline SOFA score & $10.8(9.9-11.8)$ & $8.6(6.5-10.8)$ & $11.5(10.4-12.6)$ & 0.015 \\
\hline
\end{tabular}

Values are presented as mean ( $95 \%$ confidence interval) or frequency (\%) as appropriate.

CPC, cerebral performance category; NSE, neuron-specific enolase; HDL, high-density lipoprotein; ApoA1, apolipoprotein A-1; ApoB, apolipoprotein B; APACHE, Acute Physiology and Chronic Health Evaluation; SOFA, Sequential Organ Failure Assessment.

a) Only 55 patients with available NSE results were analyzed. 
The AUROC (95\% confidence interval) of lipid profiles to predict the good CPC were as follows: total cholesterol, 0.642 (0.478 to 0.806); triglycerides, 0.604 (0.413 to 0.794); HDL, 0.799 (0.681 to 0.916 ); $A p o A 1,0.759$ (0.630 to 0.889); $A p o B, 0.595$ (0.419 to 0.770). Only HDL and ApoA1 levels allowed for discrimination be-

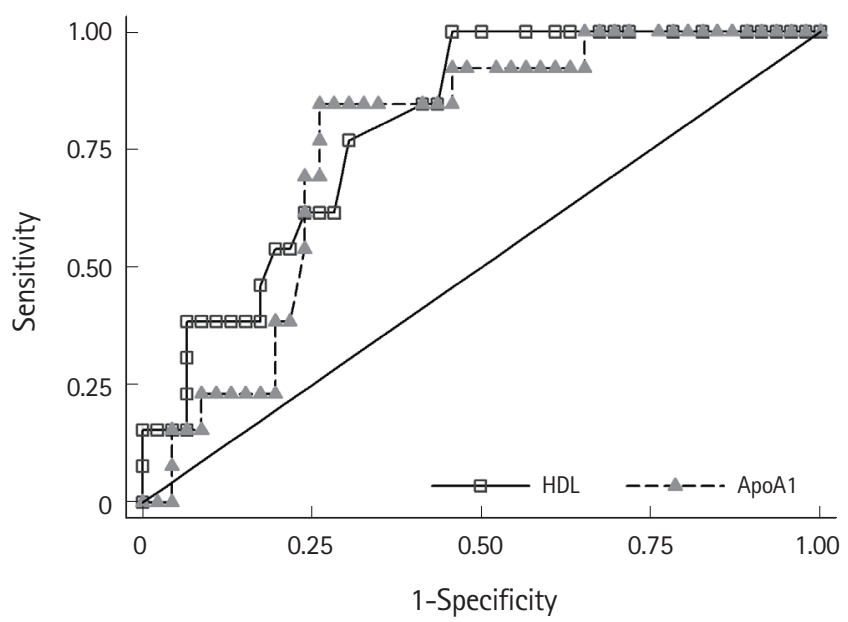

Fig. 2. Receiver operating characteristics curve of high-density lipoproteins (HDLs) and apolipoprotein A-1 (ApoA1) to predict good neurologic outcomes.

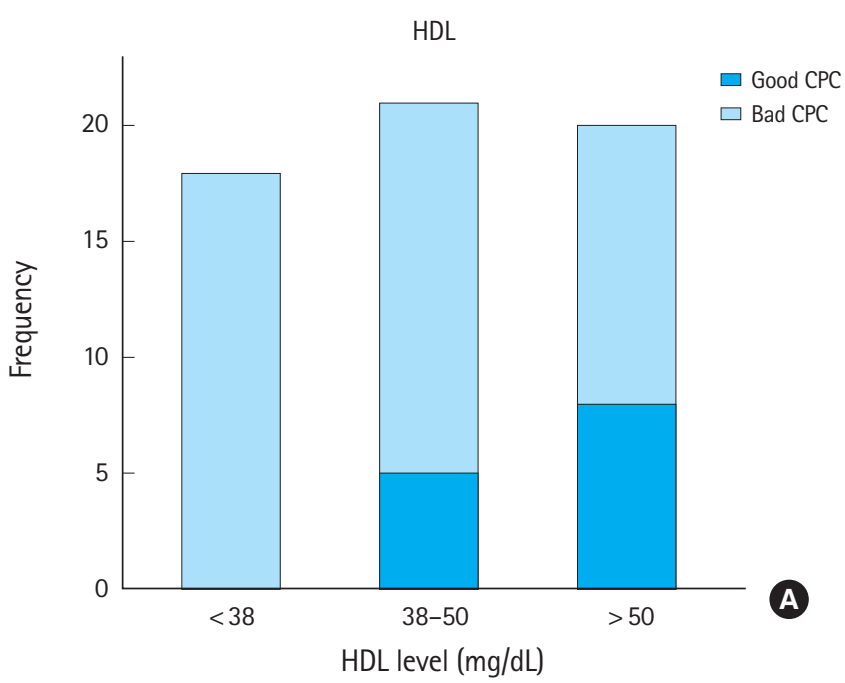

tween a good and a bad outcome (Fig. 2).

Patients were divided into tertiles, according to $\mathrm{HDL}(<38$, 3850 , and $>50 \mathrm{mg} / \mathrm{dL}$ ) and ApoA1 (<85, 85-113, and > $113 \mathrm{mg} / \mathrm{dL}$ ), and the proportion of a good CPC was significantly higher in patients with higher HDL (test for trend, $\mathrm{P}=0.003$ ) and ApoA1 (test for trend, $P=0.003$ ) levels (Fig. 3).

The diagnostic performances of HDL and ApoA1 at the tertile cutoffs are detailed in Table 2. The lowest tertiles of HDL and ApoA1 had sensitivity and negative predictive values higher than $90 \%$ for the prediction of a good CPC.

Multivariate logistic regression analysis, using significant variables in univariate analysis, was performed with backward stepwise removal. Both $\mathrm{HDL}$ and ApoA1 remained in the model finally selected (Table 3). HDL was an independent predictor for good neurologic outcome $(P=0.018)$ in model 1. However, ApoA1 was not an independent predictor $(P=0.075)$ in model 2 .

\section{DISCUSSION}

In this observational study, we found that the admission levels of HDL and ApoA1 were associated with a good discharge neurologic outcome in OHCA patients. This was demonstrated using

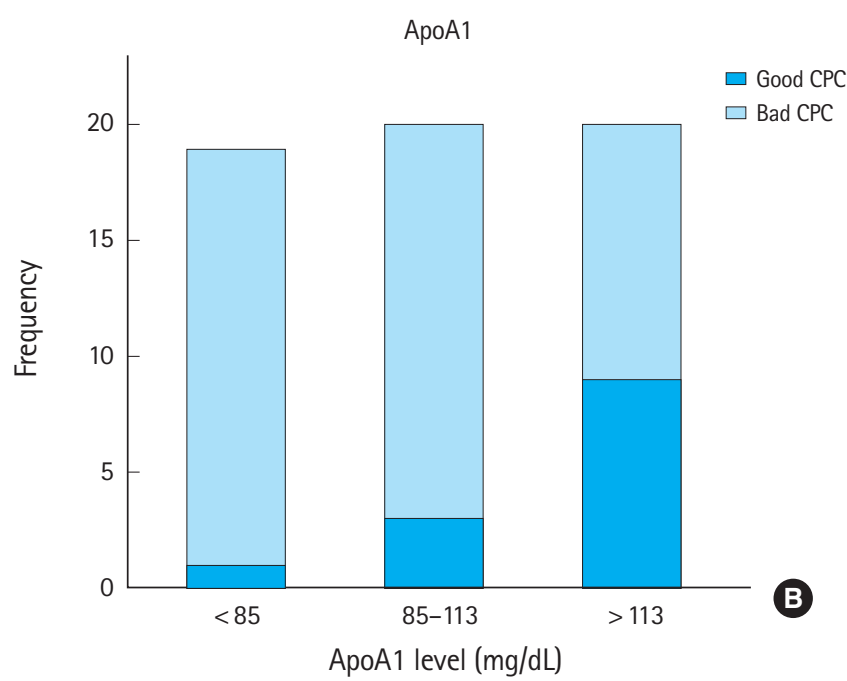

Fig. 3. Stacked bar graph representing the frequency of each neurological outcome according to tertiles of (A) high-density lipoprotein (HDL) and (B) apolipoprotein A-1 (ApoA1) levels. CPC, cerebral performance category.

Table 2. Diagnostic performances of lipid profiles to predict good neurologic outcomes

\begin{tabular}{|c|c|c|c|c|c|}
\hline Variable & Cutoff (mg/dL) & Sensitivity & Specificity & PPV & NPV \\
\hline $\mathrm{HDL}$ & 38 & $100(75.3-100)$ & $39.1(25.1-54.6)$ & 31.7 (18.1-48.1) & $100(81.5-100)$ \\
\hline HDL & 50 & $61.5(31.6-86.1)$ & 73.9 (58.9-85.7) & $40.0(19.1-63.9)$ & $87.2(72.6-95.7)$ \\
\hline ApoA1 & 85 & $92.3(64.0-99.8)$ & $39.1(25.1-54.6)$ & $30.0(16.6-46.5)$ & $94.7(74.0-99.9)$ \\
\hline ApoA1 & 113 & $69.2(38.6-90.9)$ & 76.1 (61.2-87.4) & $45.0(23.1-68.5)$ & 89.7 (75.8-97.1) \\
\hline
\end{tabular}

PPV, positive predictive value; NPV, negative predictive value; HDL, high-density lipoprotein; ApoA1, apolipoprotein A-1. 
Table 3. Multivariate logistic regression analysis to predict good neurologic outcomes ${ }^{\text {a) }}$

\begin{tabular}{lrll}
\hline Variable & Odds ratio & \multicolumn{1}{c}{$95 \% \mathrm{Cls}$} & P-value \\
\hline Model 1 & & & \\
$\quad$ Age per year & 0.92 & $0.84-1.00$ & 0.053 \\
Sex, male & 0.06 & $0.00-0.91$ & 0.043 \\
Cardiac cause & 91.73 & $3.43-2,455.47$ & 0.007 \\
HDL per 10 mg/dL increment & 3.80 & $1.25-11.52$ & 0.018 \\
Model 2 & & & \\
Age per year & 0.95 & $0.89-1.01$ & 0.078 \\
Sex, male & 0.05 & $0.00-0.69$ & 0.025 \\
Cardiac cause & 56.50 & $4.08-783.20$ & 0.003 \\
ApoA1 per 10 mg/dL increment & 1.42 & $0.97-2.08$ & 0.075 \\
\hline
\end{tabular}

$\mathrm{Cl}$, confidence interval; HDL, high-density lipoprotein; ApoA1, apolipoprotein A-1. a) Variables entered in the model include age, sex, shockable rhythm, cardiac cause, Acute Physiology and Chronic Health Evaluation II, Sequential Organ Failure Assessment score, and HDL or ApoA1.

univariate analysis, AUROC, test for trend, and multivariate analysis. To our knowledge, this is the first study that has reported the association between lipid profiles and neurologic outcomes in post-cardiac arrest patients.

As described above, the prognostic role of HDL and ApoA1 has been previously reported in patients with various acute systemic inflammatory conditions. ${ }^{6-9}$ However, the exact mechanism remains unclear. Lipoproteins can bind and neutralize bacterial substances such as endotoxins. ${ }^{11,12}$ Therefore, one possible mechanism of altered HDL and ApoA1 levels is that lipoproteins are consumed by circulating endotoxins. In a post-cardiac arrest state, endotoxemia is frequently detected and is known to be associated with hemodynamic instability. ${ }^{4,13}$ Another possible mechanism is the suppressed synthesis of ApoA1. Elevated inflammatory cytokines were consistently observed in previous studies and inflammatory cytokines are known to inhibit the synthesis of apolipoproteins. ${ }^{8,9,14,15}$

In contrast to this study, surgical patients with neurological indications had no significant hypolipidemia. ${ }^{9}$ The post-cardiac arrest state is quite different from surgical neurocritical problems such as intracranial hemorrhage. Neurosurgical patients in the previous study had low clinical severity and cytokine levels. ${ }^{9}$ However, multiple organ dysfunction is common and half of the survivors experience arterial hypotension in post-cardiac arrest patients. ${ }^{3,16}$ In our study population, clinical severity scores were high (APACHE II, 29.3 and SOFA score, 10.8) and the mean admission lactate level was $7.2 \mathrm{mmol} / \mathrm{L}$. This discrepancy in clinical severity may explain our different results. One study involving patients with ischemic stroke reported that lower HDL levels were independently associated with adverse clinical outcome, which is consistent with our findings. ${ }^{17}$ Furthermore, a preclinical study using neonatal piglets found that plasma ApoA1 levels and cerebral cortical ApoA1 ex- pression decreased after deep hypothermic circulatory arrest. ${ }^{18}$

The findings of this study may be helpful for the management of post-cardiac arrest patients in two respects. One respect concerns the prognostication of neurologic outcomes. The discriminatory capacity of $\mathrm{HDL}$ and ApoA1 to predict a positive neurologic outcome was good (AUROC, 0.799 and 0.759, respectively), and the negative predictive values of $\mathrm{HDL}$ and $\mathrm{ApoA} 1$ at the 1st tertile cutoff were 100\% and $94.7 \%$, respectively. Low HDL and ApoA1 levels may be used as an additional poor prognostic marker. The other respect is that raising $\mathrm{HDL}$ and ApoA1 levels can be a potential therapeutic option. We have reported that $4 \mathrm{~F}$, a synthetic ApoA1 mimetic peptide, could attenuate acute lung injury and improve survival in endotoxemic rats. ${ }^{19}$ This novel therapy, using synthetic HDL, is attracting researchers' attention, particularly regarding sepsis and we consider that the same approach could be applied to the post-cardiac arrest state. ${ }^{20}$

Several limitations apply to this study. First, this was a retrospective study performed in a single intensive care unit. Therefore, a selective number of patients with lipid profiles were analyzed. As a result, small numbers of patients and potential selection bias should be considered. Additionally, only 8 (17.4\%) of the bad CPC group had a cardiac cause of arrest. Low HDL and ApoA1 levels might be influenced by medical conditions other than cardiac arrest itself. For example, a higher ApoA1 is known to be associated with a lower prevalence of chronic kidney disease, and baseline creatinine levels were significantly different between the good CPC and bad CPC groups in this study. ${ }^{21}$ However, there was no difference regarding the prevalence of chronic kidney disease and baseline creatinine levels among tertiles of HDL and ApoA1. Moreover, the lowest tertile of HDL ( $<38 \mathrm{mg} / \mathrm{dL})$ group had significantly higher creatinine levels at 24 and 48 hours (Supplementary Fig. 1). The association between acute kidney injury and lipid profiles could not be addressed in this study. Supplement Tables 1 and 2 demonstrate patient characteristics according to tertiles of HDL and ApoA1. Only lactate and albumin levels were different among the tertiles of HDL. Cardiac cause and lactate, albumin, and C-reactive protein levels were different among the tertiles of ApoA1. Although we had included age, sex, shockable rhythm, cardiac cause, APACHE II, and SOFA scores in the multivariate logistic model to adjust for confounding factors, potential biases may still exist. This limitation can only be answered by a subsequent prospective study. Second, the exact pathway between systemic ischemia-reperfusion injury and lipid profiles could not be ascertained, as with previous studies.

In conclusion, admission levels of HDL and ApoA1 are associated with the neurologic outcome of patients with OHCA. Prognostic values of $\mathrm{HDL}$ and ApoA1 require further evaluation in the 
post-cardiac arrest state, as in other systemic inflammatory conditions such as sepsis.

\section{SUPPLEMENTARY MATERIALS}

Supplementary materials are available from: $h t t p s: / / d o i . o r g / 10.15441 /$ ceem.16.164.

Supplementary Table 1. Patient characteristics according to HDL tertiles

Supplementary Table 2. Patient characteristics according to ApoA1 tertiles

Supplementary Fig. 1. Serial changes of serum creatinine levels per $\mathrm{mg} / \mathrm{dL}$ stratified by (A) high-density lipoprotein $(\mathrm{mg} / \mathrm{dL}$ ) tertiles and (B) apolipoprotein A-1 (mg/dL) tertiles

\section{CONFLICT OF INTEREST}

No potential conflict of interest relevant to this article was reported.

\section{REFERENCES}

1. Centers for Disease Control and Prevention. CARES summary report: demographic and survival characteristics of OHCA [Internet]. Atlanta, GA: CARES; 2014 [cited 2017 Sep 1]. Available from: https://mycares.net/sitepages/uploads/2014/2013C ARESNationalSummaryReport.pdf.

2. Shin SD, Suh GJ, Ahn KO, Song KJ. Cardiopulmonary resuscitation outcome of out-of-hospital cardiac arrest in low-volume versus high-volume emergency departments: an observational study and propensity score matching analysis. Resuscitation 2011;82:32-9.

3. Roberts BW, Kilgannon JH, Chansky ME, et al. Multiple organ dysfunction after return of spontaneous circulation in postcardiac arrest syndrome. Crit Care Med 2013;41:1492-501.

4. Adrie C, Adib-Conquy M, Laurent I, et al. Successful cardiopulmonary resuscitation after cardiac arrest as a "sepsis-like" syndrome. Circulation 2002;106:562-8.

5. Ingelsson E, Schaefer EJ, Contois JH, et al. Clinical utility of different lipid measures for prediction of coronary heart disease in men and women. JAMA 2007;298:776-85.

6. van Leeuwen HJ, Heezius EC, Dallinga GM, van Strijp JA, Verhoef J, van Kessel KP. Lipoprotein metabolism in patients with severe sepsis. Crit Care Med 2003;31:1359-66.

7. Chien JY, Jerng JS, Yu CJ, Yang PC. Low serum level of highdensity lipoprotein cholesterol is a poor prognostic factor for severe sepsis. Crit Care Med 2005;33:1688-93.
8. Peng YS, Chen YC, Tian YC, et al. Serum levels of apolipoprotein A-I and high-density lipoprotein can predict organ failure in acute pancreatitis. Crit Care 2015;19:88.

9. Gordon BR, Parker TS, Levine DM, et al. Relationship of hypolipidemia to cytokine concentrations and outcomes in critically ill surgical patients. Crit Care Med 2001;29:1563-8.

10. Peberdy MA, Callaway CW, Neumar RW, et al. Part 9: postcardiac arrest care: 2010 American Heart Association Guidelines for Cardiopulmonary Resuscitation and Emergency Cardiovascular Care. Circulation 2010;122(18 Suppl 3):S768-86.

11. Levels JH, Abraham PR, van den Ende A, van Deventer SJ. Distribution and kinetics of lipoprotein-bound endotoxin. Infect Immun 2001;69:2821-8.

12. Flegel WA, Baumstark MW, Weinstock $C$, Berg A, Northoff $H$. Prevention of endotoxin-induced monokine release by human low- and high-density lipoproteins and by apolipoprotein A-I. Infect Immun 1993;61:5140-6.

13. Grimaldi D, Sauneuf $B$, Guivarch $E$, et al. High level of endotoxemia following out-of-hospital cardiac arrest is associated with severity and duration of postcardiac arrest shock. Crit Care Med 2015;43:2597-604.

14. Fraunberger P, Pilz G, Cremer P, Werdan K, Walli AK. Association of serum tumor necrosis factor levels with decrease of cholesterol during septic shock. Shock 1998;10:359-63.

15. Ettinger WH, Varma VK, Sorci-Thomas M, et al. Cytokines decrease apolipoprotein accumulation in medium from Hep G2 cells. Arterioscler Thromb 1994;14:8-13.

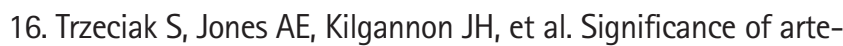
rial hypotension after resuscitation from cardiac arrest. Crit Care Med 2009;37:2895-903.

17. Putaala J, Strbian D, Mustanoja S, Haapaniemi E, Kaste M, Tatlisumak T. Functional outcome in young adult ischemic stroke: impact of lipoproteins. Acta Neurol Scand 2013;127:61-9.

18. Sheikh AM, Barrett C, Villamizar N, et al. Proteomics of cerebral injury in a neonatal model of cardiopulmonary bypass with deep hypothermic circulatory arrest. J Thorac Cardiovasc Surg 2006;132:820-8.

19. Kwon WY, Suh GJ, Kim KS, Kwak YH, Kim K. 4F, apolipoprotein Al mimetic peptide, attenuates acute lung injury and improves survival in endotoxemic rats. J Trauma Acute Care Surg 2012; 72:1576-83.

20. Morin EE, Guo L, Schwendeman A, Li XA. HDL in sepsis: risk factor and therapeutic approach. Front Pharmacol 2015;6:244.

21. Goek ON, Kottgen A, Hoogeveen RC, Ballantyne CM, Coresh J, Astor BC. Association of apolipoprotein A1 and B with kidney function and chronic kidney disease in two multiethnic population samples. Nephrol Dial Transplant 2012;27:2839-47. 